\title{
Mapping ISO 19115-1 geographic metadata standards to codemeta
}

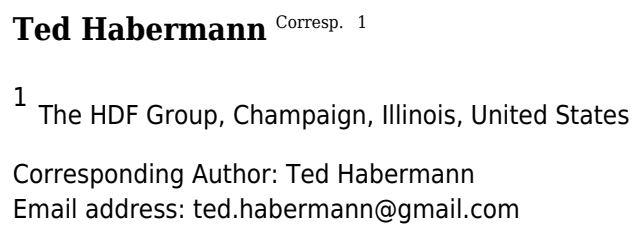

The codemeta project recently proposed a vocabulary for software metadata. ISO Technical Committee 211 has published a set of metadata standards for geographic data and many kinds of related resources, including software. In order for ISO metadata creators and users to take advantage of the codemeta recommendations, a mapping from ISO elements to the codemeta vocabulary must exist. This mapping is complicated by differences in the approaches used by ISO and codemeta, primarily a difference between hard and soft typing of metadata elements. These differences are described in detail and a mapping is proposed that includes sixty-four of the sixty-eight codemeta V2 terms. The codemeta terms have also been mapped to dialects used by twenty-one software repositories, registries and archives. The average number of terms mapped in these cases is 11.2. The disparity between these numbers reflects the fact that many of the dialects that have been mapped to codemeta are focused on citation or dependency identification and management while ISO and codemeta share additional targets that include access, use, and understanding. Addressing this broader set of use cases requires more metadata elements. 
1 Mapping ISO 19115-1 geographic metadata standards to

2 codemeta

3 Ted Habermann ${ }^{1}$

$4{ }^{1}$ The HDF Group, Champaign, Illinois, United States

5

6 Corresponding Author:

7 Ted Habermann ${ }^{1}$

81800 S. Oak Street, Suite 203, Champaign, IL, 61820-7059, United States

9 Email address: ted.habermann@gmail.com

\section{Abstract}

The codemeta project recently proposed a vocabulary for software metadata. ISO Technical Committee 211 has published a set of metadata standards for geographic data and many kinds of related resources, including software. In order for ISO metadata creators and users to take advantage of the codemeta recommendations, a mapping from ISO elements to the codemeta vocabulary must exist. This mapping is complicated by differences in the approaches used by ISO and codemeta, primarily a difference between hard and soft typing of metadata elements. These differences are described in detail and a mapping is proposed that includes sixty-four of the sixty-eight codemeta V2 terms. The codemeta terms have also been mapped to dialects used by twenty-one software repositories, registries and archives. The average number of terms mapped in these cases is 11.2. The disparity between these numbers reflects the fact that many of the dialects that have been mapped to codemeta are focused on citation or dependency identification and management while ISO and codemeta share additional targets that include access, use, and understanding. Addressing this broader set of use cases requires more metadata elements.

\section{Introduction}

The Codemeta Project (Codemeta Project) recently proposed 1) a vocabulary for documenting software, 2) mappings between metadata fields used by a broad range of software repositories, registries and archives (Codemeta Crosswalk), and 3) developed software with the purpose of facilitating automatic translation between different representations of software metadata. The vocabulary was designed to support several different software use cases, including citation, discovery, use and, to some degree, understanding.

The ISO Technical Committee 211 has developed generic metadata standards that are widely used for geographic data of many kinds. These standards were also designed as a foundation that can be built on to document many kinds of things and support many use cases (Habermann, 2018).

This paper describes a mapping between the conceptual model that underlies ISO metadata (ISO 19115-1) and Codemeta with the goal of facilitating the creation of codemeta-compliant descriptions of software that is documented using the ISO standards. The communities that developed these two metadata dialects share the important goal of comprehensive standards that address multiple use cases for many disciplines. Both groups pursue this goal by developing consensus, but the details of the processes used to develop their standards differ. ISO TC211 represents a traditional International standards body with well-defined processes, publication 
43 methods and a business model that includes costs to users for standards documents. Codemeta represents a community of volunteer practitioners with an initial set of proposed conventions open on the Web and an invitation for adoption, experimentation and evolution.

In addition to these process differences, there are also differences between the structures and implementations of these two models. These are described below with the mappings following.

\section{Dialect Coverage and Scope}

Mapping metadata for software between different schemas and dialects is an important technical goal of codemeta. This goal is supported using a crosswalk file that is maintained and contributed to in the codemeta git repository (Codemeta, 2018). This file lists the codemeta terms along with equivalents in twenty-one dialects. This crosswalk is the basis for translating content between these dialects.

A similar situation occurs in many in science communities that are trying to support multiple use cases, i.e. document, share, and trust, for datasets using multiple metadata dialects (see Gordon and Habermann, 2018). The concept of "Dialect Coverage" has come up in those studies as the amount (\%) of the concepts in a particular recommendation that a dialect includes. In the codemeta case, this is the number of codemeta concepts that can be represented in the dialects listed in the crosswalk file (Crosswalk Data). Figure 1 shows this count for each of the twentyone dialects. These counts were determined from the crosswalk file by counting the number of cells with content in each column using a spreadsheet count() function. Both versions of codemeta and ISO 19115-3 are included on the left side of this Figure as well.

The data show that the ISO dialect covers very close to all (64/68) of the codemeta concepts and the other twenty-one dialects cover an average of $11.23 / 68$, suggesting that codemeta is more similar to ISO than it is to other dialects that have done crosswalks. The difference between the ISO mapping and others is striking. It likely reflects the difference between the small number of metadata elements used for discovering and citing software (or data) and the larger number needed to be able to use it and trust it. In the current software citation landscape, this is the difference between the complete codemeta vocabulary, i.e. all metadata for code (over sixty items), and the FORCE11 Software Citation Guidelines, i.e. metadata for code citation (Smith et al., 2016) which includes only ten items.

In some cases, multiple codemeta terms are mapped to the same ISO elements. Most of this ambiguity is related to differences in the two models and approaches that are discussed in detail below. ISO elements that are mapped to more than one codemeta term are identified with * in the crosswalk tables below.

\section{Model Characteristics}

The ISO metadata standards are based on a UML model that is harmonized across all standards developed and managed by the committee. The model is built around classes and attributes that describe the structure of the standards and the relationships among objects. ISO 19115-1 includes thirteen top-level classes that provide details on identification, content, constraints, distribution, quality, usage, reference systems, spatial representation and several other areas.

The ISO standard includes a scope element at the root of each record that gives the type of resource described by the metadata. The default scope is dataset, but other options include: aggregate, application, attribute, attributeType, collection, collectionHardware, 
86 collectionSession, coverage, dimensionGroup, document, feature, featureType, fieldSession,

87 initiative, metadata, model, nonGeographicDataset, otherAggregate, platformSeries, product,

88 productionSeries, propertyType, repository, sample, sensor, sensorSeries, series, service,

89 software, tile, transferAggregate (see Habermann, 2018). Mapping the codemeta vocabulary to

90 the ISO standard is an initial step toward defining the content that could be included in ISO

91 metadata records that describe software and applications, i.e. those where the scope is software.

92 The most commonly used representation of the ISO standards is XML (ISO 19115-3). ISO

93 XPaths uniquely identify metadata content and follow the structure of the UML model, with

94 levels in the XML alternating between objects (with types) and properties. This results in XML

95 that is "striped" like the XML representation of the Resource Description Framework (RDF)

96 (W3C, 2014), i.e. role/type/role/type/content. Types generally start with two uppercase letters

97 (MD, CI, ...) that indicate the UML package that they are defined in (metadata, citation, ...)

98 followed by an underscore (MD_, CI_,...). Properties (termed roles in this discussion) are in

99 lower camel case.

100 A significant benefit of the striped XML is that properties can be defined with abstract objects

101 that can share properties while being instantiated with different types. For example, the ISO

102 CI_Party object is abstract and includes name and contactInfo properties. It is extended and

103

104

105 specialized by CI_Individual and CI_Organisation objects which inherit name and contactInfo properties add properties that are relevant for people and organizations, e.g. organizations can include individuals, logos, and position names. This approach also facilitates reuse by allowing standard objects (e.g. people, organizations, or citations) to be referenced using links rather than repetitive content (https://geo-ide.noaa.gov/wiki/index.php?title=ISO_Components).

Another benefit of this approach in ISO is the same as that in the schema.org case communities can extend object definitions when necessary and, in the ISO case, the resulting extended objects fit naturally into the ISO XML representation. This approach is similar to the schema extension model used in codemeta to add properties deemed important by the codemeta

113 community to the more general SoftwareSourceCode schema that is also a specialization of the schema.CreativeWork schema.

114

The namespace for each element in the XML is identified using a standard namespace prefix $(\mathrm{mdb}, \mathrm{cit}, . .$.$) . Asterisks are used in the XPaths to indicate locations where several objects can be$ used. For example, mdb:identificationInfo/*/ indicates that either mdb:MD_DataIdentification or srv:SV_ServiceIdentification objects can occur in that location.

118 A simplified notation is introduced for paths through the UML conceptual model in this document that includes only the role names and no information that is specific to the XML representation. For example, the XPath

/mdb:MD_Metadata/mdb:identificationInfo/mri:MD_DataIdentification/mri:resourceSpecificUsa ge/mri:MD_Usage/mri:identifiedIssues/cit:CI_Citation/cit:onlineResource/cit:CI_OnlineResourc e/cit:linkage is replaced by the concept path: identificationInfo.resourceSpecificUsage.identifiedIssues.onlineResource.linkage. These simplified "concept paths" improve readability and emphasize equivalences between codemeta and ISO in the conceptual space. Specific XPaths can be constructed from these concept paths when necessary to implement translation of existing ISO content to codemeta representations. The reverse translation is not unique.

Codemeta specifies a vocabulary rather than a structural model. It includes properties from 131 (Crosswalk Data). These schemas exist in a schema.org hierarchy which is similar in many ways 
132 to the ISO structure. SoftwareApplication and SoftwareSourceCode schemas are both

133 specializations of the Thing > CreativeWork schema. Codemeta extends these schemas (in

134 codemeta.SoftwareSourceCode) with several properties that lack clear equivalents in

135 schema.org.

136 Hard Types and Soft Types

137 All standards and vocabularies need to make choices between hard or soft typing of objects

138 they are describing. Hard typing requires specific names for items and is the only choice

139 available in implementations where names alone can be used to distinguish between items, e.g.

140 codemeta. For example, if publication and revision dates are required for complete descriptions

141 of a resource, hard typed representations would include two items: e.g. publicationDate and

142 revisionDate. Soft Typing can be used in dialects which support item attributes as well as values,

143 e.g. XML. In that case, these two dates would be represented with the same name (XML

144 element) and distinguished by a type attribute: $<$ datetype="publication" $>$ and

145 <datetype="revision">.

146 The difference between these two approaches emerges as the dialects evolve. Hard types

147 evolve by adding new elements to the underlying model, i.e. adding creationDate (or some other

148 type of date) when it becomes apparent that it is needed, and unambiguous definitions of those

149 elements. Soft types evolve by adding items to the shared vocabulary of date types, typically a

150 codelist or thesaurus.

151

152

The critical difference between hard and soft types boils down to differences in governance models and change tolerance. In communities that use hard types, members must be tolerant to

153

154

155

156

157

158

159

160

161

162

163

164

165 changes in the models and, typically, changes in tooling built on them. Communities that use soft typing must have mechanisms for sharing and evolving vocabularies, typically control bodies or rules.

The ISO model is soft-typed and the Codemeta model is hard-typed. Table 2 lists some of the documentation concepts that illustrate the contrast between these approaches. The first row shows the differences in how dates are treated. The codemeta vocabulary includes four types of dates listed in the second column of Table 2. If other date types are required to describe software, maybe dateDeprecated for example, new terms would be found in schema.org or added to the vocabulary to address those needs. The ISO approach involves a single date concept and a codelist that includes sixteen options, shown in the third column of Table 2. That codelist is designed to be extended by communities with other needs without impacting the structure of the standard. In this example, the date type codelist already includes the term "deprecated".

\section{Citations}

Connecting users to resources is one of the most important roles of metadata. It is also one of the most ubiquitous. Several classes of citations are important:

169 1. Citation to the resource being described in the metadata (Resource Citation). The role of these citations is to provide guidance on how the resource being described should be cited and there is only one of these in each metadata record.

2. Citations to related resources (Related Resource Citation). These are generic references to some other resource and generally include information about the relationship between the resource being described and the related resource. See, for example, the RelatedIdentifier

174 element in the DataCite metadata schema (DataCite Metadata Working Group, 2017) which 
175 includes relatedIdentifierType and relationType attributes as additional information.

176 3. Citations to other, typically specific, resources (Specific Resource Citations). For example, the

177 ISO object that describes data processing includes a citation in the role of softwareReference that

178 specifically provides a reference to software used in the processing.

179 Other examples of these citation types are included in the following discussion.

180

181

182

183

184

185

186

187

188

189

190

191

192

193

194

195

196

197

198

199

200

201

202

203

204

205

206

207

208

209

210

211

212

213

\section{Additional Documentation} dataQualityInfo.standaloneQualityReport.

\section{Codemeta Citations}

\section{Distribution} (function=information).

ISO 19115-1 includes all three types of citations:

- The Resource Citation is unique and occurs at a specific location in the conceptual model: identificationInfo.citation $(\mathrm{XPath}=$ /mdb:MD_Metadata/mdb:identificationInfo/*/mri:citation/cit:CI_Citation).

- Related Resource Citations also occur at a specific location in the model, identificationInfo.associatedResource $(\mathrm{XPath}=$ /mdb:MD_Metadata/mdb:identificationInfo/*/mri:associatedResource/mri:MD_Associate dResource/mri:name/cit:CI_Citation along with two codelists (associationType and initiativeType) that provide information about how the resource is associated.).

- Specific Resource Citations occur in a number of locations in the ISO model as part of specific classes. For example, citations to additional documentation occur at identificationInfo.additionalDocumentation and citations to quality reports occur at

All ISO Citations include elements of traditional citations to books or papers e.g. title, authors (people or organizations in many roles), dates (many types), series information, page numbers, etc., as well as identifiers (ISSN, ISBN, and other types) and URLs with titles, descriptions and types. The XPaths to these items from each ISO citation root are shown in Table 3.

Codemeta includes twenty-six terms that represent resources that are related to or support the use of the software being described. These terms have several different types (Text, URL, Text or URL, CreativeWork, CreativeWork or URL, Computer Language or text, ...). In the mappings below, these terms are mapped to the ISO citations. The specific types can be described by adding the paths in Table 3 to the concept or XPaths.

Many of the distribution systems for geographic data described by ISO metadata include repositories (generally called archives or data centers) that manage and preserve data while providing on-going support for users. ISO metadata standards accommodate approaches to resource distribution with or without descriptions of repositories (termed distributors) and each repository can provide several URLs (transferOptions) for each resource. These onlineResources can have any of the functions included in the CI_OnLineFunctionCode codelist in Table 2. The most common online functions are download and information and these are used in the mappings to indicate direct access to the resource (function=download) or information about the resource 
215 The codemeta vocabulary includes many items that are intended to help users use and 216 understand the software described in the metadata. In the ISO standards, these items can be

217 described in two ways: as associated resources (identificationInfo.associatedResource) or as

218 additional documentation (identificationInfo.additionalDocumentation). I have chosen the later

219 in these cases. In dialects without specific citations, e.g. Datacite, these would be referred to as

220

221

222

223

224

225

226

227

228

229

230 Metadata Working Group, 2017).

One important goal of codemeta is to enable authors to cite software that is used to store, process, analyze, and visualize the data and model results that they use in their work. Increasing citations from the scientific literature is large part of this goal, but there are also significant opportunities to improve the completeness of dataset metadata by citing software. This is generally done as part of the provenance or lineage section of the metadata. The ISO standards provide several specific resource citations for citing software, including:

- resourceLineage.processStep.processingInformation.algorithm.citation,

- resourceLineage.processStep.processingInformation.softwareReference, and

- resourceLineage.processStep.processingInformation.documentation.

\section{Mappings}

The mappings between codemeta and ISO are presented here in a series of tables that correspond to the source schema.org schemas used in order to provide some structure that may help clarify the relationships and improve understanding. The process of creating the mappings involved three steps: 1) obvious connections, i.e. description -> identificationInfo.abstract, or name -> identificationInfo.citation.title, 2) more complicated connections like those discussed above, and 3) matching intended types as closely as possible, i.e. codemeta terms that were intended to be identifiers were mapped to ISO identifier codes (identifier -> identificationInfo.citation.identifier.code) and those that were intended to be URLs were mapped to ISO linkages (downloadUrl -> distributionInfo.transferOptions.onLine[function='download'].linkage). This process is, of course, more subjective than objective, and the resulting mappings reflect experience authoring the ISO standards and working with them in multiple contexts over the last decade.

The mappings include the property names, types, and descriptions from the codemeta vocabulary, conceptual paths for the ISO items (ISO 19115-1), and XPaths from the standard XML representation (ISO 19115-3). The conceptual paths are provided here in lieu of the ISO definitions for simplicity. The complete ISO conceptual model with definitions is available in an HTML view (ISO Conceptual Model). In some cases, multiple codemeta terms are mapped to single ISO elements, as described in the Additional Documentation section above. These cases are marked with * in the tables.

These mappings are also available in machine-readable forms (Habermann, figshare, 2018 and Habermann, codemeta, 2018).

\section{Schema:Person}

The schema.Person schema provides a vocabulary for properties of people. In the ISO standards, people and organizations are both referred to as parties and names can be given as any combination of individual names, organization names, or positions. This mapping includes seven items listed in Table 4. 
258

259

260

261

262

263

264

265

266

267

268

269

270

271

272

273

274

275

276

277

278

279

280

281

282

283

284

285

286

287

288

289

290

291

292

293

294

295

296

\section{Schema:Thing}

The schema.Thing schema provides a vocabulary for properties of the most generic type of item. In the context of codemeta, this item is the resource described by the metadata which is software. In ISO 19115-1, properties related to the identification of the resource being described are in the identificationInfo section and many of the properties are included in the citation to that resource. As described above, these properties (title, identifier, and link) are included in all citations in the ISO model. This mapping includes six items listed in Table 5.

\section{Schema:Thing.CreativeWork}

The Thing.CreativeWork schema provides a vocabulary for the most generic kind of creative work, including books, movies, photographs, software programs, etc. This mapping includes twenty-four items listed in Table 6.

\section{Schema:Thing.CreativeWork.SoftwareSourceCode}

The Thing.CreativeWork.SoftwareSourceCode schema provides a vocabulary for describing computer programming source code. This mapping includes four items listed in Table 7.

\section{Codemeta:SoftwareSourceCode}

The codemeta:SoftwareSourceCode schema extends Thing.CreativeWork.SoftwareSourceCode with terms created by the codemeta project. This mapping includes ten items listed in Table 8.

\section{Schema:Thing.CreativeWork.SoftwareApplication}

The Thing.CreativeWork.SoftwareApplication schema provides a vocabulary for describing a software application. This mapping includes fifteen items listed in Table 9.

\section{Conclusions}

The ISO metadata standards were originally developed by ISO Technical Committee 211 to serve as the standard and structured part of the documentation needed to discover, access, use, and understand datasets. The standards acknowledge that they are generic, and they include several mechanisms for extension to address specific needs of communities that build on the standards. The generic nature of these standards is reflected in the breadth of the codelist that can be used to describe the scope of a particular metadata record (see list in Model Characteristics Section above and Habermann, 2018).

The codemeta project recently proposed over sixty terms that can be used in metadata for software. This recommendation provides a framework that provides insight into what ISO metadata for software might contain. The ISO metadata standards include several elements that directly cite software, e.g. the processingInformation.softwareReference or algorithm.citation elements, and the primary purpose of the mappings proposed here is to support ISO users that want to 1) express software metadata in a dialect that they are familiar with and 2) to facilitate translation of software metadata written using ISO standards to codemeta-compliant JSON-LD.

Note that the purpose is different than the primary purpose of the crosswalks proposed on the codemeta project site which is to facilitate automated translations between different JSON-LD vocabularies and RDF. Adding an ISO crosswalk to the codemeta framework was the original 
297

298

299

300

301

302

303

304

305

306

307

308

309

310

311

312

313

314

315

316

317

318

319

320

321

322

323

324

325

326

327

328

329

330

331

332

333

334

335

336

337

338

339

goal of this work, but the differences identified and described here are significant enough to make that impossible. This translation may be facilitated with an XSLT built specifically for that purpose, like the one created by Peroni et al., 2012, but that is beyond the scope of this initial comparison.

The process of creating a mapping between these two representations surfaced some differences that complicate the mapping. Some of these differences are related to hard and soft typing used in the two models and others are related to increased flexibility that is required in a generic standard like ISO for documenting citations, distribution channels, and related resources.

The differences in approach described here probably apply to many mappings from XML to RDF representations. Peroni et al., 2012 identified and discussed some similar challenges when mapping between JATS XML and SPAR Ontologies. They attributed these differences to "differing philosophical viewpoints for XML and RDF", then described two reasons that XML element names are ambiguous when used in isolation: attributes and hierarchical structure. They suggest that the JATS standard (and by implication all XML representations) is "deliberately vague" and that the hierarchical structure of XML is "not formalized and implicitly lives outside the XML schema of the language". It is certainly true that XML element names can be ambiguous without associated attributes and hierarchical structure but ignoring those methods of providing meaning in XML and then stating that elements alone are ambiguous does not contribute to understanding differences between how these two approaches describe resources.

I examine these differences in more detail and offer an explanation that is related to different approaches to defining types: XML can use attributes and structure to provide information about types and meaning while RDF must rely on unambiguous and shared definitions of terms. To use the case described in Peroni et al., 2012, the JATS element "article" is definitely ambiguous. That is why the definition of the element includes an attribute called article-type to clarify the type of the article being described. Details of the importance of attributes for semantics in XML is described in detail by Seligy, 2018 along with potential problems that result from ignoring attribute values.

Peroni et al, 2012 also acknowledge the requirement for unambiguous definitions that are shared across communities. They state that "A cornerstone of the Semantic Web is the use of open published ontologies to give precise and universally available definitions to terms, so that RDF statements, whatever else they are, are unambiguous in their meaning." While this may be a goal of ontology development efforts, many of the definitions currently used in codemeta and schema.org (shown in Tables 5-9) are unclear, non-unique, and ambiguous.

ISO mappings are proposed for sixty-four of the sixty-eight codemeta V2 terms. These mappings can be used to create codemeta-compliant metadata from existing stores of ISO metadata and to add codemeta compliant software citations in the future. This compares to an average of 11.2 mappings for other dialects included in the codemeta crosswalk. This disparity reflects the use cases targeted by the dialects. Many of the dialects that have been mapped to codemeta are focused on citation or dependency identification and management while ISO and codemeta share additional targets that include access, use, and understanding.

\section{Acknowledgements}

Thanks to Matt Jones, Carl Boettiger, Dennis Walworth, and Melissa Harrison for helpful comments and discussion of the initial draft of this paper. 
340 References

341 Codemeta Crosswalk, https://codemeta.github.io/crosswalk/, accessed 2018-08-20.

342 Crosswalk Data,

343 https://github.com/codemeta/codemeta/blob/8027db72f1c2b956453b38cc4711e498bdaa46ce/cro

344 sswalk.csv, accessed 2018-08-20.

345 Codemeta Project, https://codemeta.github.io, accessed 2018-10-01.

346 DataCite Metadata Working Group. (2017). DataCite Metadata Schema Documentation for the

347 Publication and Citation of Research Data. Version 4.1. DataCite e.V. 10.5438/0014.

348 Gordon, Sean, and Ted Habermann. "The Influence of Community Recommendations on

349 Metadata Completeness." Ecological Informatics, vol. 43, Jan. 2018, pp. 38-51. Crossref,

350 doi:10.1016/j.ecoinf.2017.09.005.

351 Habermann, Ted. "Metadata Life Cycles, Use Cases and Hierarchies." Geosciences, vol. 8, no. 5,

352 May 2018, p. 179. Crossref, doi:10.3390/geosciences8050179.

353 Habermann, Ted. MappingTables_ISO19115-1ToCodemeta.csv., 2018, figshare. Dataset.

354 doi:10.6084/m9.figshare.7430282.v1

355 Habermann, Ted. Codemeta mapping to ISO 19115, 2018,

356 https://github.com/codemeta/codemeta/blob/master/crosswalks/ISO 19115-1.csv, accessed

357 2019-01-04.

358 ISO 19115-1, ISO 19115-1:2014, Geographic information -- Metadata -- Part 1: Fundamentals,

359 https://www.iso.org/standard/53798.html, accessed 2018-10-01.

360 ISO 19115-3, ISO/TS 19115-3:2016, Geographic information -- Metadata -- Part 3: XML schema

361 implementation for fundamental concepts, https://www.iso.org/standard/32579.html, accessed

362 2018-10-01.

363 ISO Conceptual Model - HTML View,

364 http://www.isotc211.org/hmmg/HTML/ConceptualModels/, accessed 2018-10-01.

365 Peroni, S., D.A. Lapeyre, and D. Shotton, 2012, From Markup to Linked Data: Mapping NISO

366 JATS v1.0 to RDF using the SPAR (Semantic Publishing and Referencing) Ontologies, Journal

367 Article Tag Suite Conference (JATS-Con) Proceedings 2012 [Internet]. Bethesda (MD):

368 National Center for Biotechnology Information (US),

369 https://www.ncbi.nlm.nih.gov/books/NBK100491/, accessed 2018-10-01.

370 RDF, https://en.wikipedia.org/wiki/Resource Description_Framework, accessed 2018-12-05.

371 Seligy, M., 2018, Listen up! Is it time for systems to start hearing what attributes are saying?, ,

372 Journal Article Tag Suite Conference (JATS-Con) Proceedings 2018 [Internet]. Bethesda (MD):

373 National Center for Biotechnology Information (US),

374 https://www.ncbi.nlm.nih.gov/books/NBK493053/, accessed 2018-10-01.

375 Smith, Arfon M., Daniel S. Katz, Kyle E. Niemeyer, FORCE11 Software Citation Working

376 Group, "Software Citation Principles." PeerJ Computer Science, vol. 2, Sept. 2016, p.

377 e86. Crossref, doi:10.7717/peerj-cs.86.

378 W3C, RDF 1.1. XML Syntax Recommendation, 2014, https://www.w3.org/TR/rdf-syntax-

379 grammar/\#section-Syntax-intro, accessed 2018-08-21 
Figure 1

Coverage of codemeta concepts in multiple dialects.

The average number of codemeta concepts covered by twenty-one dialects is 11.2. The ISO dialect covers sixty-four of sixty-eight codemeta concepts.

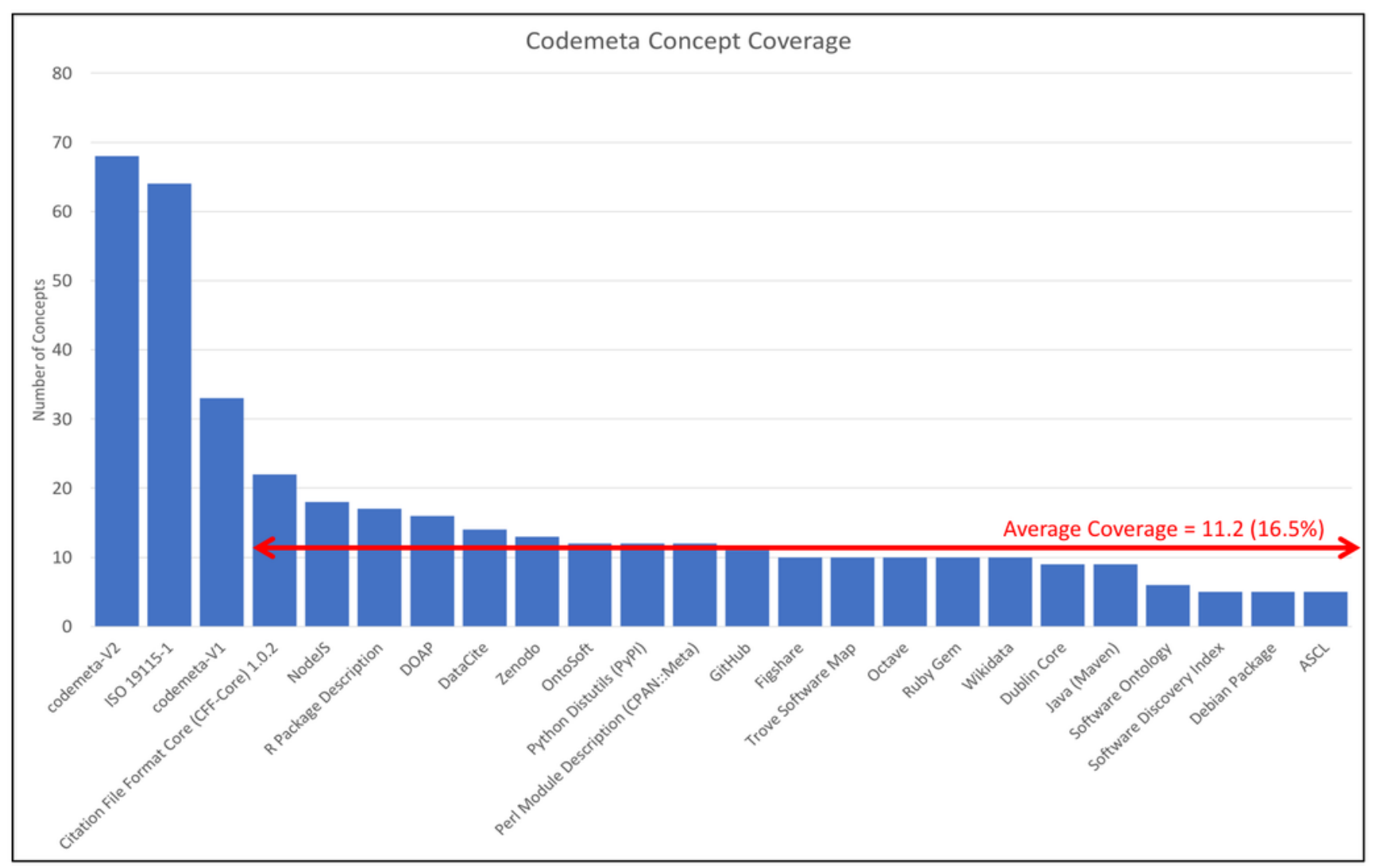




\section{Table $\mathbf{1}$ (on next page)}

Schema.org schemas and item counts for codemeta vocabulary. 
1

\begin{tabular}{|l|c|l|c|}
\hline Source & \#Terms & Source & \#Terms \\
\hline schema:CreativeWork & 24 & schema:Thing & 6 \\
\hline schema:SoftwareApplication & 15 & schema:SoftwareSourceCode & 4 \\
\hline codemeta:SoftwareSourceCode & 10 & schema (not mapped) & 2 \\
\hline schema:Person & 7 & & \\
\hline
\end{tabular}

2 


\section{Table 2 (on next page)}

Codemeta hard types and related ISO codelists. 


\begin{tabular}{|c|c|c|}
\hline Item & Codemeta items & ISO $19115-1$ codelist values* \\
\hline Dates & $\begin{array}{l}\text { embargoDate dateCreated } \\
\text { dateModified datePublished }\end{array}$ & $\begin{array}{l}\text { CI_DateTypeCode: creation publication } \\
\text { revision expiry lastUpdate lastRevision } \\
\text { nextUpdate unavailable inForce adopted } \\
\text { deprecated superseded validityBegins } \\
\text { validityExpires released distribution }\end{array}$ \\
\hline $\begin{array}{l}\text { People and } \\
\text { Organizations }\end{array}$ & $\begin{array}{l}\text { author contributor creator } \\
\text { copyrightHolder editor funder } \\
\text { producer provider publisher sponsor } \\
\text { affiliation }\end{array}$ & $\begin{array}{l}\text { CI_RoleCode: resourceProvider custodian } \\
\text { owner user distributor originator } \\
\text { pointOfContact principalInvestigator } \\
\text { processor publisher author sponsor coAuthor } \\
\text { collaborator editor mediator rightsHolder } \\
\text { contributor funder stakeholder maintainer }\end{array}$ \\
\hline $\begin{array}{l}\text { Online } \\
\text { Resource } \\
\text { Types }\end{array}$ & $\begin{array}{l}\text { buildInstructions contIntegration } \\
\text { issueTracker readme id identifier } \\
\text { downloadUrl installUrl } \\
\text { codeRepository relatedLink sameAs } \\
\text { url }\end{array}$ & $\begin{array}{l}\text { CI_OnLineFunctionCode: download } \\
\text { information offlineAccess order search } \\
\text { completeMetadata browseGraphic upload } \\
\text { emailService browsing fileAccess }\end{array}$ \\
\hline Associations & supportingData & $\begin{array}{l}\text { DS_AssociationTypeCode: crossReference } \\
\text { largerWorkCitation partOfSeamlessDatabase } \\
\text { stereoMate isComposedOf collectiveTitle } \\
\text { series dependency revisionOf }\end{array}$ \\
\hline Keyword & $\begin{array}{l}\text { Keywords programmingLanguage } \\
\text { applicationCategory } \\
\text { applicationSubCategory }\end{array}$ & $\begin{array}{l}\text { MD_KeywordTypeCode: discipline place } \\
\text { stratum temporal theme dataCentre } \\
\text { featureType instrument platform process } \\
\text { project service product subTopicCategory } \\
\text { taxon }\end{array}$ \\
\hline
\end{tabular}




\section{Table 3(on next page)}

Relative XPaths to titles, identifiers, and URLs in ISO citations. 


\begin{tabular}{|l|l|}
\hline Item & xPath from CI_Citation \\
\hline Title & cit:CI_Citation/cit:title/gco:CharacterString (concept $=$ title) \\
\hline Identifier & $\begin{array}{l}\text { cit:CI_Citation/cit:identifier/mcc:MD_Identifier/mcc:code/gco:CharacterString (concept }= \\
\text { identifier.code) }\end{array}$ \\
\hline URL & $\begin{array}{l}\text { cit:CI_Citation/cit:onlineResource/cit:CI_OnlineResource/cit:linkage/gco:CharacterString } \\
\text { (concept }=\text { onlineResource.linkage) }\end{array}$ \\
\hline
\end{tabular}

2 


\section{Table 4 (on next page)}

Mapping of codemeta terms from the schema.Person schema to ISO 19115-1 and ISO 19115-3. 


\begin{tabular}{|c|c|c|c|c|}
\hline Property & Type & Description & ISO 19115-1 & ISO 19115-3 \\
\hline address & $\begin{array}{l}\text { PostalAddress or } \\
\text { Text }\end{array}$ & Physical address of the item. & party.contactlnfo.address.deliveryPoint & $\begin{array}{l}\text { cit:party/cit:CI_Organisation/cit:contactInfo/cit: } \\
\text { CI_Contact/cit:address/cit:CI_Address/cit:delive } \\
\text { ryPoint/gco:CharacterString }\end{array}$ \\
\hline affiliation & Text & $\begin{array}{l}\text { An organization that this person is affiliated with. For example, a } \\
\text { school/university }\end{array}$ & party.name* & $\begin{array}{l}\text { cit:party/cit:CI_Organisation/cit:name/gco:Char } \\
\text { acterString }\end{array}$ \\
\hline email & Text & Email address & $\begin{array}{l}\text { party.contactInfo.address. } \\
\text { electronicMailAddress }\end{array}$ & $\begin{array}{l}\text { cit:party/cit:CI_Organisation/cit:contactInfo/cit: } \\
\text { CI_Contact/cit:address/cit:CI_Address/cit:electr } \\
\text { onicMailAddress/gco:CharacterString }\end{array}$ \\
\hline familyName & Text & $\begin{array}{l}\text { Family name. In the U.S., the last name of an Person. This can be used along } \\
\text { with givenName instead of the name property. }\end{array}$ & party.name* & $\begin{array}{l}\text { cit:party/cit:CI_Individual/cit:name/gco:Charact } \\
\text { erString }\end{array}$ \\
\hline givenName & Text & $\begin{array}{l}\text { Given name. In the U.S., the first name of a Person. This can be used along with } \\
\text { familyName instead of the name property }\end{array}$ & party.name* & $\begin{array}{l}\text { cit:party/cit:Cl_Individual/cit:name/gco:Charact } \\
\text { erString }\end{array}$ \\
\hline identifier & URL & URL identifer, ideally an ORCID ID for individuals, a FundRef ID for funders & party.partyldentifier.code & $\begin{array}{l}\text { cit:party/cit:CI_Organisation/cit:partyldentifier/ } \\
\text { mcc:MD_Identifier/mcc:code/gco:CharacterStri } \\
\text { ng }\end{array}$ \\
\hline name & Text & $\begin{array}{l}\text { The name of an Organization, or if separate given and family names cannot be } \\
\text { resolved, for a Person }\end{array}$ & party.name* & $\begin{array}{l}\text { cit:party/cit:CI_Organisation/cit:name/gco:Char } \\
\text { acterString }\end{array}$ \\
\hline
\end{tabular}




\section{Table 5 (on next page)}

Mapping of codemeta terms from the schema.Thing schema to ISO 19115-1 and ISO 19115-3. 


\begin{tabular}{|c|c|c|c|c|}
\hline Property & Type & Description & ISO 19115-1 & ISO 19115-3 \\
\hline description & Text & A description of the item. & identificationInfo.abstract & $\begin{array}{l}\text { /mdb:MD_Metadata/mdb:identificationlnfo/*/ } \\
\text { mri:abstract/gco:CharacterString }\end{array}$ \\
\hline identifier & $\begin{array}{l}\text { PropertyValue or } \\
\text { URL }\end{array}$ & $\begin{array}{l}\text { The identifier property represents any kind of identifier for any kind of Thing, } \\
\text { such as ISBNs, GTIN codes, UUIDs etc. Schema.org provides dedicated } \\
\text { properties for representing many of these, either as textual strings or as URL } \\
\text { (URI) links. See background notes for more details. }\end{array}$ & identificationInfo.citation.identifier.code & $\begin{array}{l}\text { /mdb:MD_Metadata/mdb:identificationInfo/*/ } \\
\text { mri:citation/cit:Cl_Citation/cit:identifier/mcc:M } \\
\text { D__dentifier/mcc:code }\end{array}$ \\
\hline name & Text & The name of the item (software, Organization) & identificationInfo.citation.title & $\begin{array}{l}\text { /mdb:MD_Metadata/mdb:identificationlnfo/*/ } \\
\text { mri:citation/cit:CI_Citation/cit:title/gco:Characte } \\
\text { rString }\end{array}$ \\
\hline relatedLink & URL & A link related to this object, e.g. related web pages & $\begin{array}{l}\text { identification!nfo.citation.onlineResource } \\
\text { [function='information']* }\end{array}$ & $\begin{array}{l}\text { /mdb:MD_Metadata/mdb:identificationInfo/*/ } \\
\text { mri:citation/cit:CI_Citation/cit:onlineResource/ci } \\
\text { t:CI_OnlineResource/mdb:MD_Metadata/mdb:i } \\
\text { dentificationInfo/*/mri:citation/cit:CI_Citation/c } \\
\text { it:onlineResource/cit:CI_OnlineResource[gmd:fu } \\
\text { nction//gmd:_l_OnLineFunctionCode,'informatio } \\
\text { n'] /cit:linkage }\end{array}$ \\
\hline sameAs & URL & $\begin{array}{l}\text { URL of a reference Web page that unambiguously indicates the item's identity. } \\
\text { E.g. the URL of the item's Wikipedia page, Wikidata entry, or official website. }\end{array}$ & $\begin{array}{l}\text { identification!nfo.citation.onlineResource } \\
\text { [function='information']* }\end{array}$ & $\begin{array}{l}\text { /mdb:MD_Metadata/mdb:identificationInfo/*/ } \\
\text { mri:citation/cit:CI_Citation/cit:onlineResource/ci } \\
\text { t:CI_OnlineResource/mdb:MD_Metadata/mdb:i } \\
\text { dentificationlnfo/*/mri:citation/cit:CI_Citation/c } \\
\text { it:onlineResource/cit:CI_OnlineResource[gmd:fu } \\
\text { nction//gmd:CI_OnLineFunctionCode,'informatio } \\
\text { n']/cit:linkage }\end{array}$ \\
\hline url & URL & URL of the item. & $\begin{array}{l}\text { identification!nfo.citation.onlineResource } \\
\text { [function='download']* }\end{array}$ & $\begin{array}{l}\text { /mdb:MD_Metadata/mdb:identificationInfo/*/ } \\
\text { mri:citation/cit:CI_Citation/cit:onlineResource/ci } \\
\text { t:CI_OnlineResource[gmd:function/gmd:CI_OnLi } \\
\text { neFunctionCode,'download']/cit:linkage }\end{array}$ \\
\hline
\end{tabular}




\section{Table 6(on next page)}

Mapping of codemeta terms from the Thing.CreativeWork schema to ISO 19115-1 and ISO 19115-3. 


\begin{tabular}{|c|c|c|c|c|}
\hline Property & Type & Description & ISO 19115-1 & ISO 19115-3 \\
\hline author & $\begin{array}{l}\text { Organization or } \\
\text { Person }\end{array}$ & $\begin{array}{l}\text { The author of this content or rating. Please note that author is special in that } \\
\text { HTML } 5 \text { provides a special mechanism for indicating authorship via the rel tag. } \\
\text { That is equivalent to this and may be used interchangeably. }\end{array}$ & $\begin{array}{l}\text { identificationInfo.citation. } \\
\text { citedResponsibleParty[role='author'].party.name or } \\
\text { identificationlnfo.citation. } \\
\text { citedResponsibleParty[role='originator'].party.name }\end{array}$ & $\begin{array}{l}\text { /mdb:MD_Metadata/mdb:identificationInfo/*/ } \\
\text { mri:citation/cit:CI_Citation/cit:citedResponsibleP } \\
\text { arty/cit:CI_Responsibility[cit:role/cit:CI_RoleCod } \\
\text { e='author'] or } \\
\text { /mdb:MD_Metadata/mdb:identificationInfo/*/ } \\
\text { mri:citation/cit:CI_Citation/cit:citedResponsibleP } \\
\text { arty/cit:CI_Responsibility[cit:role/cit:CI_RoleCod } \\
\text { e='originator'] }\end{array}$ \\
\hline citation & $\begin{array}{l}\text { CreativeWork or } \\
\text { URL }\end{array}$ & $\begin{array}{l}\text { A citation or reference to another creative work, such as another publication, } \\
\text { web page, scholarly article, etc. }\end{array}$ & identificationInfo.associatedResource.name* & $\begin{array}{l}\text { /mdb:MD_Metadata/mdb:identificationlnfo/*/ } \\
\text { mri:associatedResource/mri:MD_AssociatedReso } \\
\text { urce/mri:name/cit:Cl_Citation }\end{array}$ \\
\hline contributor & $\begin{array}{l}\text { Organization or } \\
\text { Person }\end{array}$ & A secondary contributor to the CreativeWork or Event. & $\begin{array}{l}\text { identificationInfo.citation.citedResponsibleParty } \\
\text { [not(role='author' or role=''principallnvestigator' or } \\
\text { role='originator')].party.name* }\end{array}$ & $\begin{array}{l}\text { /mdb:MD_Metadata/mdb:identificationInfo/*/ } \\
\text { mri:citation/cit:CI_Citation/cit:citedResponsibleP } \\
\text { arty/cit:CI_Responsibility[not(cit:role/cit:CI_Role } \\
\text { Code='author' or } \\
\text { cit:role/cit:CI_RoleCode='principallnvestigator' } \\
\text { or cit:role/cit:CI_RoleCode='originator')] } \\
\text { /cit:party/*cit:name }\end{array}$ \\
\hline $\begin{array}{l}\text { copyright } \\
\text { Holder }\end{array}$ & $\begin{array}{l}\text { Organization or } \\
\text { Person }\end{array}$ & The party holding the legal copyright to the CreativeWork. & $\begin{array}{l}\text { identificationInfo.resourceConstraints.reference. } \\
\text { citedResponsibleParty }\end{array}$ & $\begin{array}{l}\text { /mdb:MD_Metadata/mdb:identificationlnfo/*/ } \\
\text { mri:resourceConstraints/mco:MD_LegalConstrai } \\
\text { nts/mco:reference/cit:CI_Citation/cit:citedRespo } \\
\text { nsibleParty/cit:CI_Responsibility }\end{array}$ \\
\hline $\begin{array}{l}\text { copyright } \\
\text { Year }\end{array}$ & Number & $\begin{array}{l}\text { The year during which the claimed copyright for the CreativeWork was first } \\
\text { asserted. }\end{array}$ & $\begin{array}{l}\text { identificationInfo.resourceConstraints.reference. } \\
\text { date[dateType='publication'].date }\end{array}$ & $\begin{array}{l}\text { /mdb:MD_Metadata/mdb:identificationlnfo/*/ } \\
\text { mri:resourceConstraints/mco:MD_LegalConstrai } \\
\text { nts/mco:reference/cit:CI_Citation/cit:date/cit:Cl } \\
\text { _Date[cit:dateType/cit:CI_DateTypeCode='public } \\
\text { ation']/cit:dateType }\end{array}$ \\
\hline creator & $\begin{array}{l}\text { Organization or } \\
\text { Person }\end{array}$ & $\begin{array}{l}\text { The creator/author of this CreativeWork. This is the same as the Author } \\
\text { property for CreativeWork. }\end{array}$ & $\begin{array}{l}\text { identificationlnfo.citation.citedResponsibleParty } \\
\text { [role='author'].party.name or } \\
\text { identificationInfo.citation.citedResponsibleParty[role='o } \\
\text { riginator'].party.name* }\end{array}$ & $\begin{array}{l}\text { /mdb:MD_Metadata/mdb:identificationInfo/*/ } \\
\text { mri:citation/cit:CI_Citation/cit:citedResponsibleP } \\
\text { arty/cit:CI_Responsibility[cit:role/cit:CI_RoleCod } \\
\text { e='author'] or } \\
\text { /mdb:MD_Metadata/mdb:identificationInfo/*/ } \\
\text { mri:citation/cit:CI_Citation/cit:citedResponsibleP } \\
\text { arty/cit:CI_Responsibility[cit:role/cit:CI_RoleCod } \\
\text { e='originator'] }\end{array}$ \\
\hline $\begin{array}{l}\text { date } \\
\text { Created }\end{array}$ & Date or DateTime & $\begin{array}{l}\text { The date on which the CreativeWork was created or the item was added to a } \\
\text { DataFeed. }\end{array}$ & $\begin{array}{l}\text { identificationInfo.citation.date[dateType='creation']. } \\
\text { date* }\end{array}$ & $\begin{array}{l}\text { /mdb:MD_Metadata/mdb:identificationInfo/*/ } \\
\text { mri:citation/cit:CI_Citation/cit:date/cit:Cl_Date[c } \\
\text { it:dateType/cit:CI_DateTypeCode='creation']/cit: } \\
\text { date/gco:DateTime }\end{array}$ \\
\hline $\begin{array}{l}\text { date } \\
\text { Modified }\end{array}$ & Date or DateTime & $\begin{array}{l}\text { The date on which the CreativeWork was most recently modified or when the } \\
\text { item's entry was modified within a DataFeed. }\end{array}$ & $\begin{array}{l}\text { identificationInfo.citation.date[dateType='revision']. } \\
\text { date* }\end{array}$ & $\begin{array}{l}\text { /mdb:MD_Metadata/mdb:identificationInfo/*/ } \\
\text { mri:citation/cit:CI_Citation/cit:date/cit:CI_Date[c } \\
\text { it:dateType/cit:CI_DateTypeCode='revision']/cit: } \\
\text { date/gco:DateTime }\end{array}$ \\
\hline
\end{tabular}




\begin{tabular}{|c|c|c|c|c|}
\hline Property & Type & Description & ISO 19115-1 & ISO 19115-3 \\
\hline $\begin{array}{l}\text { date } \\
\text { Published }\end{array}$ & Date & Date of first broadcast/publication. & $\begin{array}{l}\text { identificationInfo.citation.date[dateType='publication']. } \\
\text { date* }\end{array}$ & $\begin{array}{l}\text { /mdb:MD_Metadata/mdb:identificationInfo/*/ } \\
\text { mri:citation/cit:CI_Citation/cit:date/cit:CI_Date[c } \\
\text { it:dateType/cit:CI_DateTypeCode='publication']/ } \\
\text { cit:date/gco:Date }\end{array}$ \\
\hline editor & Person & Specifies the Person who edited the CreativeWork. & $\begin{array}{l}\text { identificationInfo.citation.citedResponsibleParty } \\
\text { [role='editor'].party.name* }\end{array}$ & $\begin{array}{l}\text { /mdb:MD_Metadata/mdb:identificationInfo/*/ } \\
\text { mri:citation/cit:CI_Citation/cit:citedResponsibleP } \\
\text { arty/cit:Cl_Responsibility[cit:role/cit:Cl_RoleCod } \\
\text { e='editor'] }\end{array}$ \\
\hline encoding & MediaObject & $\begin{array}{l}\text { A media object that encodes this CreativeWork. This property is a synonym } \\
\text { for associatedMedia. Supersedes encodings. }\end{array}$ & & \\
\hline fileFormat & Text or URL & $\begin{array}{l}\text { Media type, typically MIME format (see IANA site) of the content e.g. } \\
\text { application/zip of a SoftwareApplication binary. In cases where a } \\
\text { CreativeWork has several media type representations, 'encoding' can be used } \\
\text { to indicate each MediaObject alongside particular fileformat information. } \\
\text { Unregistered or niche file formats can be indicated instead via the most } \\
\text { appropriate URL, e.g. defining Web page or a Wikipedia entry. }\end{array}$ & $\begin{array}{l}\text { identificationInfo.resourceFormat. } \\
\text { formatSpecificationCitation }\end{array}$ & $\begin{array}{l}\text { /mdb:MD_Metadata/mdb:identificationlnfo/mri: } \\
\text { MD_Dataldentification/mri:resourceFormat/mrd } \\
\text { :MD_Format/mrd:formatSpecificationCitation/ci } \\
\text { t:Cl_Citation }\end{array}$ \\
\hline funder & $\begin{array}{l}\text { Organization or } \\
\text { Person }\end{array}$ & $\begin{array}{l}\text { A person or organization that supports (sponsors) something through some } \\
\text { kind of financial contribution. }\end{array}$ & $\begin{array}{l}\text { identificationInfo.citation.citedResponsibleParty } \\
\text { [role'='funder'].party.name* }\end{array}$ & $\begin{array}{l}\text { /mdb:MD_Metadata/mdb:identificationInfo/*/ } \\
\text { mri:citation/cit:CI_Citation/cit:citedResponsibleP } \\
\text { arty/cit:Cl_Responsibility[cit:role/cit:Cl_RoleCod } \\
\text { e='funder'] }\end{array}$ \\
\hline hasPart & CreativeWork & $\begin{array}{l}\text { Indicates a CreativeWork that is (in some sense) a part of this CreativeWork. } \\
\text { Reverse property isPartof }\end{array}$ & $\begin{array}{l}\text { identificationlnfo.associatedResource } \\
\text { [associationType='isComposedOf'].name* }\end{array}$ & $\begin{array}{l}\text { /mdb:MD_Metadata/mdb:identificationInfo/*/ } \\
\text { mri:associatedResource/mri:MD_AssociatedReso } \\
\text { urce[mri:associationType/mri:DS_AssociationTyp } \\
\text { eCode='isComposedOf']/mri:name/cit:CI_Citatio } \\
\text { n }\end{array}$ \\
\hline $\begin{array}{l}\text { isAccessible } \\
\text { ForFree }\end{array}$ & Boolean & A flag to signal that the publication is accessible for free. & $\begin{array}{l}\text { distributionInfo.distributionFormat.formatDistributor. } \\
\text { distributionOrderProcess.fees }\end{array}$ & $\begin{array}{l}\text { /mdb:MD_Metadata/mdb:distributionlnfo/mrd: } \\
\text { MD_Distribution/mrd:distributionFormat/mrd:M } \\
\text { D_Format/mrd:formatDistributor/mrd:MD_Distr } \\
\text { ibutor/mrd:distributionOrderProcess/mrd:MD_S } \\
\text { tandardOrderProcess/mrd:fees }\end{array}$ \\
\hline isPartof & CreativeWork & $\begin{array}{l}\text { Indicates a CreativeWork that this CreativeWork is (in some sense) part of. } \\
\text { Reverse property hasPart }\end{array}$ & $\begin{array}{l}\text { identificationInfo.associatedResource } \\
\text { [associationType='LargerWorkCitation'].name* }\end{array}$ & $\begin{array}{l}\text { /mdb:MD_Metadata/mdb:identificationInfo/*/ } \\
\text { mri:associatedResource/mri:MD_AssociatedReso } \\
\text { urce[mri:associationType/mri:DS_AssociationTyp } \\
\text { eCode='LargerWorkCitation']/mri:name/cit:CI_Ci } \\
\text { tation }\end{array}$ \\
\hline keywords & Text & $\begin{array}{l}\text { Keywords or tags used to describe this content. Multiple entries in a keywords } \\
\text { list are typically delimited by commas. }\end{array}$ & $\begin{array}{l}\text { identificationInfo.descriptiveKeywords[type='theme']. } \\
\text { Keyword* }\end{array}$ & $\begin{array}{l}\text { /mdb:MD_Metadata/mdb:identificationlnfo/*/ } \\
\text { mri:descriptiveKeywords/mri:MD_Keywords[mri: } \\
\text { type/mri:MD_KeywordTypeCode='theme']/mri:k } \\
\text { eyword/gco:CharacterString }\end{array}$ \\
\hline license & $\begin{array}{l}\text { CreativeWork or } \\
\text { URL }\end{array}$ & A license document that applies to this content, typically indicated by URL. & identificationInfo.resourceConstraints.reference & $\begin{array}{l}\text { /mdb:MD_Metadata/mdb:identificationlnfo/mri: } \\
\text { MD_Dataldentification/mri:resourceConstraints/ } \\
\text { mco:MD_LegalConstraints/mco:reference/cit:CI_ } \\
\text { Citation }\end{array}$ \\
\hline
\end{tabular}




\begin{tabular}{|c|c|c|c|c|}
\hline Property & Type & Description & ISO 19115-1 & ISO 19115-3 \\
\hline position & Integer or Text & $\begin{array}{l}\text { The position of an item in a series or sequence of items. (While schema.org } \\
\text { considers this a property of CreativeWork, it is also the way to indicate } \\
\text { ordering in any list (e.g. the Authors list). By default arrays are unordered in } \\
\text { JSON-LD }\end{array}$ & & \\
\hline producer & $\begin{array}{l}\text { Organization or } \\
\text { Person }\end{array}$ & $\begin{array}{l}\text { The person or organization who produced the work (e.g. music album, movie, } \\
\text { tv/radio series etc.). }\end{array}$ & $\begin{array}{l}\text { identificationInfo.citation.citedResponsibleParty } \\
\text { [role='creator'].party.name* }\end{array}$ & $\begin{array}{l}\text { /mdb:MD_Metadata/mdb:identificationlnfo/*/ } \\
\text { mri:citation/cit:CI_Citation/cit:citedResponsibleP } \\
\text { arty/cit:CI_Responsibility[cit:role/cit:CI_RoleCod } \\
\text { e='creator']/cit:party/** }\end{array}$ \\
\hline provider & $\begin{array}{l}\text { Organization or } \\
\text { Person }\end{array}$ & $\begin{array}{l}\text { The service provider, service operator, or service performer; the goods } \\
\text { producer. Another party (a seller) may offer those services or goods on behalf } \\
\text { of the provider. A provider may also serve as the seller. Supersedes carrier. }\end{array}$ & $\begin{array}{l}\text { identificationInfo.pointOfContact } \\
\text { [role='pointOfContact'].party.name* }\end{array}$ & $\begin{array}{l}\text { /mdb:MD_Metadata/mdb:identificationlnfo/srv: } \\
\text { SV_Serviceldentification/mri:pointOfContact// } \\
\text { cit:CI_Responsibility[cit:role/cit:CI_RoleCode='pr } \\
\text { ovider']/cit:party/* }\end{array}$ \\
\hline publisher & $\begin{array}{l}\text { Organization or } \\
\text { Person }\end{array}$ & The publisher of the creative work. & $\begin{array}{l}\text { identificationInfo.citation.citedResponsibleParty } \\
\text { [role='publisher'].party.name* }\end{array}$ & $\begin{array}{l}\text { /mdb:MD_Metadata/mdb:identificationInfo/*/ } \\
\text { mri:citation/cit:CI_Citation/cit:citedResponsibleP } \\
\text { arty/cit:CI_Responsibility[cit:role/cit:CI_RoleCod } \\
\text { e='publisher']/cit:party/* }\end{array}$ \\
\hline sponsor & $\begin{array}{l}\text { Organization or } \\
\text { Person }\end{array}$ & $\begin{array}{l}\text { A person or organization that supports a thing through a pledge, promise, or } \\
\text { financial contribution. e.g. a sponsor of a Medical Study or a corporate } \\
\text { sponsor of an event. }\end{array}$ & $\begin{array}{l}\text { identificationInfo.citation.citedResponsibleParty } \\
\text { [role='sponsor'].party.name* }\end{array}$ & $\begin{array}{l}\text { /mdb:MD_Metadata/mdb:identificationlnfo/*/ } \\
\text { mri:citation/cit:CI_Citation/cit:citedResponsibleP } \\
\text { arty/cit:CI_Responsibility[cit:role/cit:CI_RoleCod } \\
\text { e='sponsor']/cit:party/* }\end{array}$ \\
\hline version & Number or Text & The version of the CreativeWork embodied by a specified resource. & identificationInfo.citation.edition & $\begin{array}{l}\text { /mdb:MD_Metadata/mdb:identificationlnfo/mri } \\
\text { MD_Dataldentification/mri:citation/cit:CI_Citatic } \\
\text { n/cit:edition/gco:CharacterString }\end{array}$ \\
\hline
\end{tabular}




\section{Table 7 (on next page)}

Mapping of codemeta terms from the Thing.CreativeWork.SoftwareSourceCode schema to ISO 19115-1 and ISO 19115-3. 


\begin{tabular}{|c|c|c|c|c|}
\hline Property & Type & Description & ISO 19115-1 & ISO 19115-3 \\
\hline $\begin{array}{l}\text { Code } \\
\text { Repository }\end{array}$ & URL & $\begin{array}{l}\text { Link to the repository where the un-compiled, human readable code and } \\
\text { related code is located (SVN, github, CodePlex). }\end{array}$ & 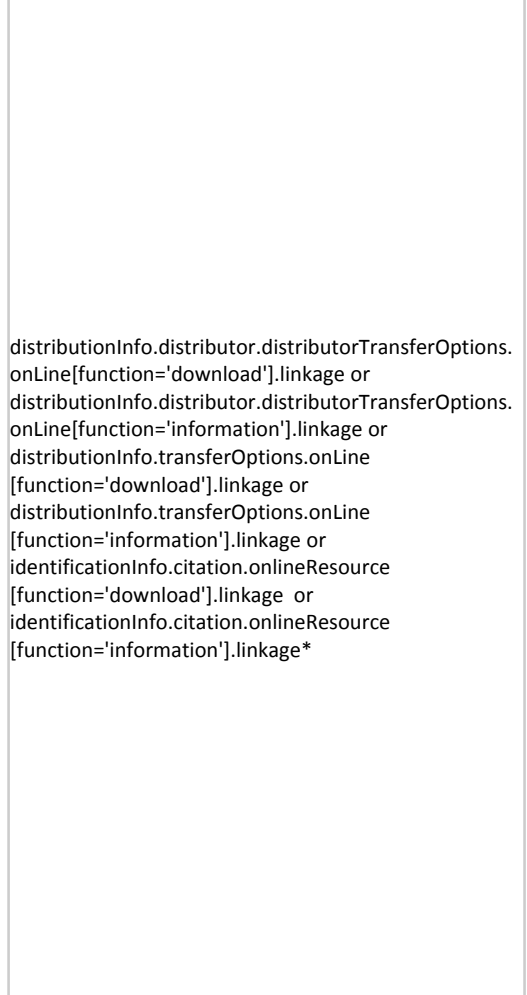 & 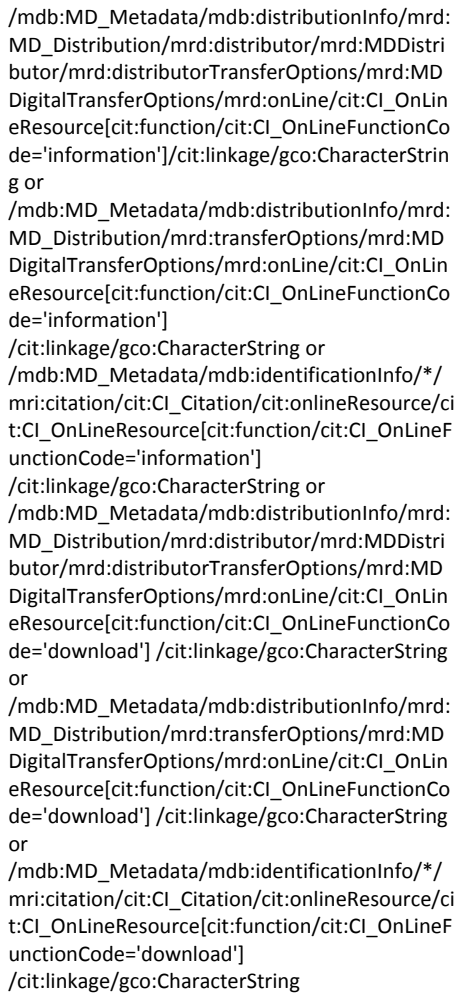 \\
\hline $\begin{array}{l}\text { Programmin } \\
\text { gLanguage }\end{array}$ & $\begin{array}{l}\text { Computer } \\
\text { Language or Text }\end{array}$ & The computer programming language. & $\begin{array}{l}\text { identificationInfo.descriptiveKeywords[type='theme']. } \\
\text { keyword* }\end{array}$ & $\begin{array}{l}\text { /mdb:MD_Metadata/mdb:identificationlnfo/*/ } \\
\text { mri:descriptiveKeywords/mri:MD_Keywords[mri } \\
\text { :type/mri:MD_KeywordTypeCode='theme']/mri: } \\
\text { keyword/gco:CharacterString }\end{array}$ \\
\hline $\begin{array}{l}\text { Runtime } \\
\text { Platform }\end{array}$ & Text & $\begin{array}{l}\text { Runtime platform or script interpreter dependencies (Example - Java v1, } \\
\text { Python2.3, Net Framework 3.0). Supersedes runtime. }\end{array}$ & identificationInfo.environmentDescription* & $\begin{array}{l}\text { /mdb:MD_Metadata/mdb:identificationlnfo/mri } \\
\text { :MD_Dataldentification/mri:environmentDescri } \\
\text { ption/gco:CharacterString }\end{array}$ \\
\hline $\begin{array}{l}\text { Target } \\
\text { Product }\end{array}$ & $\begin{array}{l}\text { Software } \\
\text { Application }\end{array}$ & $\begin{array}{l}\text { Target Operating System / Product to which the code applies. If applies to } \\
\text { several versions, just the product name can be used. }\end{array}$ & identificationInfo.associatedResource.name* & $\begin{array}{l}\text { /mdb:MD_Metadata/mdb:identificationlnfo/*/ } \\
\text { mri:associatedResource/mri:MD_AssociatedRes } \\
\text { ource/mri:name/cit:CI_Citation }\end{array}$ \\
\hline
\end{tabular}




\section{Table 8 (on next page)}

Mapping of codemeta terms from the codemeta.SoftwareSourceCode schema to ISO 19115-1 and ISO 19115-3. 


\begin{tabular}{|c|c|c|c|c|}
\hline Property & Type & Description & ISO 19115-1 & ISO 19115-3 \\
\hline $\begin{array}{l}\text { build } \\
\text { Instructions }\end{array}$ & URL & link to installation instructions/documentation & identificationInfo.additionalDocumentation ${ }^{*}$ & $\begin{array}{l}\text { /mdb:MD_Metadata/mdb:identificationlnfo/*/ } \\
\text { mri:additionalDocumentation/cit:CI_Citation }\end{array}$ \\
\hline $\begin{array}{l}\text { cont } \\
\text { Integration }\end{array}$ & URL & link to continuous integration service & identificationInfo.additionalDocumentation ${ }^{*}$ & $\begin{array}{l}\text { /mdb:MD_Metadata/mdb:identificationlnfo/*/ } \\
\text { mri:additionalDocumentation/cit:CI_Citation }\end{array}$ \\
\hline $\begin{array}{l}\text { development } \\
\text { Status }\end{array}$ & Text & $\begin{array}{l}\text { Description of development status, e.g. Active, inactive, supsended. See } \\
\text { repostatus.org }\end{array}$ & identificationInfo.status & $\begin{array}{l}\text { /mdb:MD_Metadata/mdb:identificationlnfo/*/ } \\
\text { mri:status/mcc:MD_ProgressCode }\end{array}$ \\
\hline $\begin{array}{l}\text { embargo } \\
\text { Date }\end{array}$ & Date & $\begin{array}{l}\text { Software may be embargoed from public access until a specified date (e.g. } \\
\text { pending publication, } 1 \text { year from publication) }\end{array}$ & $\begin{array}{l}\text { identificationInfo.citation.date[dateType='released']. } \\
\text { date }^{*}\end{array}$ & $\begin{array}{l}\text { /mdb:MD_Metadata/mdb:identificationlnfo/*/ } \\
\text { mri:citation/cit:CI_Citation/cit:date/cit:CI_Date[ } \\
\text { cit:dateType/cit:CI_DateTypeCode='released']/ci } \\
\text { t:date/gco:DateTime }\end{array}$ \\
\hline funding & Text & Funding source (e.g. specific grant) & identificationInfo.associatedResource" & $\begin{array}{l}\text { /mdb:MD_Metadata/mdb:identificationlnfo/*/ } \\
\text { mri:associatedResource/mri:MD_AssociatedRes } \\
\text { ource/mri:name/cit:CI_Citation }\end{array}$ \\
\hline issueTracker & URL & link to software bug reporting or issue tracking system & $\begin{array}{l}\text { identificationInfo.resourceSpecific Usage. } \\
\text { identifiedlssues.onlineResource.linkage }\end{array}$ & $\begin{array}{l}\text { /mdb:MD_Metadata/mdb:identificationlnfo/*/ } \\
\text { mri:resourceSpecificUsage/mri:MD_Usage/mri:i } \\
\text { dentifiedlssues/cit:CI_Citation }\end{array}$ \\
\hline maintainer & Person & $\begin{array}{l}\text { Individual responsible for maintaining the software (usually includes an email } \\
\text { contact address) }\end{array}$ & identificationInfo.pointOfContact & $\begin{array}{l}\text { /mdb:MD_Metadata/mdb:identificationlnfo/*/ } \\
\text { mri:pointOfContact }\end{array}$ \\
\hline readme & URL & link to software Readme file & identificationInfo.additionalDocumentation" & $\begin{array}{l}\text { /mdb:MD_Metadata/mdb:identificationlnfo/*/ } \\
\text { mri:additionalDocumentation/cit:CI_Citation }\end{array}$ \\
\hline $\begin{array}{l}\text { reference } \\
\text { Publication }\end{array}$ & ScholarlyArticle & An academic publication related to the software. & identificationInfo.additionalDocumentation* & $\begin{array}{l}\text { /mdb:MD_Metadata/mdb:identificationlnfo/*/ } \\
\text { mri:additionalDocumentation/cit:CI_Citation }\end{array}$ \\
\hline $\begin{array}{l}\text { software } \\
\text { Suggestions }\end{array}$ & $\begin{array}{l}\text { SoftwareSourceC } \\
\text { ode }\end{array}$ & Optional dependencies, e.g. for optional features, code development, etc & identificationInfo.additionalDocumentation ${ }^{*}$ & $\begin{array}{l}\text { /mdb:MD_Metadata/mdb:identificationlnfo/*/ } \\
\text { mri:additionalDocumentation/cit:CI_Citation }\end{array}$ \\
\hline
\end{tabular}




\section{Table 9 (on next page)}

Mapping of Codemeta terms from the schema:Thing.CreativeWork.SoftwareApplication schema to ISO 19115-1 and 19115-3. 


\begin{tabular}{|c|c|c|c|c|}
\hline Property & Type & Description & ISO 19115-1 & ISO 19115-3 \\
\hline $\begin{array}{l}\text { application } \\
\text { Category }\end{array}$ & Text or URL & Type of software application, e.g. 'Game, Multimedia'. & $\begin{array}{l}\text { identificationInfo.descriptiveKeywords[type='the } \\
\text { me'].keyword* }\end{array}$ & $\begin{array}{l}\text { /mdb:MD_Metadata/mdb:identificationlnfo/*/ } \\
\text { mri:descriptiveKeywords/mri:MD_Keywords[mri } \\
\text { :type/mri:MD_KeywordTypeCode='theme']/mri: } \\
\text { keyword/gco:CharacterString }\end{array}$ \\
\hline $\begin{array}{l}\text { application } \\
\text { SubCategory }\end{array}$ & Text or URL & Subcategory of the application, e.g. 'Arcade Game'. & $\begin{array}{l}\text { identificationlnfo.descriptiveKeywords[type='the } \\
\text { me'].keyword* }\end{array}$ & $\begin{array}{l}\text { /mdb:MD_Metadata/mdb:identificationlnfo/*/ } \\
\text { mri:descriptiveKeywords/mri:MD_Keywords[mri } \\
\text { :type/mri:MD_KeywordTypeCode='theme']/mri: } \\
\text { keyword/gco:CharacterString }\end{array}$ \\
\hline $\begin{array}{l}\text { download } \\
\text { Url }\end{array}$ & URL & If the file can be downloaded, URL to download the binary. & $\begin{array}{l}\text { distributionInfo.distributor.distributorTransferO } \\
\text { ptions.onLine[function='download'].linkage or } \\
\text { distributionInfo.transferOptions.onLine[function } \\
=\text { 'download'].linkage or } \\
\text { identificationInfo.citation.onlineResource[functi } \\
\text { on='download'].linkage }\end{array}$ & 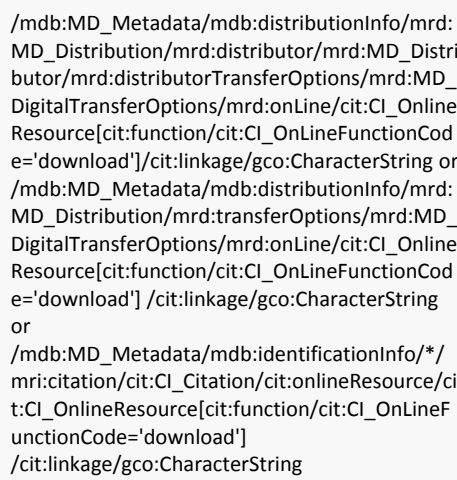 \\
\hline filesize & Text & $\begin{array}{l}\text { Size of the application / package (e.g. 18MB). In the absence of a unit (MB, KB } \\
\text { etc.), KB will be assumed. }\end{array}$ & $\begin{array}{l}\text { distributionInfo.transferOptions.transferSize or } \\
\text { distributionInfo.distributionFormat.formatDistri } \\
\text { butor.distributorTransferOptions.transferSize or } \\
\text { distributionInfo.distributor.distributorTransferO } \\
\text { ptions.transferSize }\end{array}$ & $\begin{array}{l}\text { /mdb:MD_Metadata/mdb:distributionInfo/mrd: } \\
\text { MD_Distribution/mrd:transferOptions/mrd:MD } \\
\text { DigitalTransferOptions/mrd:transferSize/gco:Rea } \\
\text { I or } \\
\text { /mdb:MD_Metadata/mdb:distributionlnfo/mrd: } \\
\text { MD_Distribution/mrd:distributionFormat/mrd: } \\
\text { MD_Format/mrd:formatDistributor/mrd:MD_Di } \\
\text { stributor/mrd:distributorTransferOptions/mrd: } \\
\text { MD_DigitalTransferOptions/mrd:transferSize/gc } \\
\text { o:Real or } \\
\text { /mdb:MD_Metadata/mdb:distributionInfo/mrd: } \\
\text { MD_Distribution/mrd:distributor/mrd:MD_Distri } \\
\text { butor/mrd:distributorTransferOptions/mrd:MD_- } \\
\text { DigitalTransferOptions/mrd:transferSize/gco:Rea } \\
\text { I }\end{array}$ \\
\hline installurl & URL & URL at which the app may be installed, if different from the URL of the item. & $\begin{array}{l}\text { distributionInfo.distributor.distributorTransferO } \\
\text { ptions.onLine[function='download'].linkage or } \\
\text { distributionInfo.transferOptions.onLine[function } \\
=\text { 'download'].linkage or } \\
\text { identificationInfo.citation.onlineResource[functi } \\
\text { on='download'].linkage }\end{array}$ & $\begin{array}{l}\text { /mdb:MD_Metadata/mdb:distributionlnfo/mrd: } \\
\text { MD_Distribution/mrd:distributor/mrd:MD_Distri } \\
\text { butor/mrd:distributorTransferOptions/mrd:MD_ } \\
\text { DigitalTransferOptions/mrd:onLine/cit:CI_Online } \\
\text { Resource[cit:function/cit:CI_OnLineFunctionCod } \\
\text { e='download']/cit:linkage/gco:CharacterString or } \\
\text { /mdb:MD_Metadata/mdb:distributionInfo/mrd: }\end{array}$ \\
\hline
\end{tabular}




\begin{tabular}{|c|c|c|c|c|}
\hline Property & Type & Description & ISO 19115-1 & ISO 19115-3 \\
\hline & & & & $\begin{array}{l}\text { MD_Distribution/mrd:transferOptions/mrd:MD } \\
\text { DigitalTransferOptions/mrd:onLine/cit:CI_Online } \\
\text { Resource[cit:function/cit:CI_OnLineFunctionCod } \\
\text { e='download']/cit:linkage/gco:CharacterString or or } \\
\text { /mdb:MD_Metadata/mdb:identificationlnfo/*/ } \\
\text { mri:citation/cit:CI_Citation/cit:onlineResource/c } \\
\text { t:CI_OnlineResource[cit:function/cit:CI_OnLineF } \\
\text { unctionCode='download']/cit:linkage/gco:Charac } \\
\text { terString }\end{array}$ \\
\hline $\begin{array}{l}\text { memory } \\
\text { Requiremen } \\
\text { ts }\end{array}$ & Text or URL & Minimum memory requirements. & identificationInfo.environmentDescription* & $\begin{array}{l}\text { /mdb:MD_Metadata/mdb:identificationlnfo/mri } \\
: \text { :MD_Dataldentification/mri:environmentDescrip } \\
\text { tion/gco:CharacterString }\end{array}$ \\
\hline $\begin{array}{l}\text { operating } \\
\text { System }\end{array}$ & Text & Operating systems supported (Windows 7, OSX 10.6, Android 1.6). & identificationInfo.environmentDescription* & $\begin{array}{l}\text { /mdb:MD_Metadata/mdb:identificationlnfo/mri } \\
: \text { :MD_Dataldentification/mri:environmentDescrip } \\
\text { tion/gco:CharacterString }\end{array}$ \\
\hline permissions & Text & $\begin{array}{l}\text { Permission(s) required to run the app (for example, a mobile app may require } \\
\text { full internet access or may run only on wifi). }\end{array}$ & $\begin{array}{l}\text { identificationInfo.resourceConstraints or } \\
\text { identificationInfo.resourceConstraints.reference. } \\
\text { onlineResource.linkage }\end{array}$ & $\begin{array}{l}\text { /mdb:MD_Metadata/mdb:identificationlnfo/*/ } \\
\text { mri:resourceConstraints/mco:MD_LegalConstrai } \\
\text { nts or } \\
\text { /mdb:MD_Metadata/mdb:identificationlnfo/mri } \\
\text { :MD_Dataldentification/mri:resourceConstraints } \\
\text { /mco:MD_LegalConstraints/mco:reference/cit:C } \\
\text { _Citation/cit:onlineResource/cit:CI_OnlineResou } \\
\text { rce/cit:linkage }\end{array}$ \\
\hline $\begin{array}{l}\text { processor } \\
\text { Requiremen } \\
\text { ts }\end{array}$ & Text & Processor architecture required to run the application (e.g. IA64). & identificationInfo.environmentDescription* & $\begin{array}{l}\text { /mdb:MD_Metadata/mdb:identificationlnfo/mri } \\
\text { :MD_Dataldentification/mri:environmentDescrip } \\
\text { tion/gco:CharacterString }\end{array}$ \\
\hline $\begin{array}{l}\text { release } \\
\text { Notes }\end{array}$ & Text or URL & Description of what changed in this version. & identificationInfo.additionalDocumentation* & $\begin{array}{l}\text { /mdb:MD_Metadata/mdb:identificationlnfo/*/ } \\
\text { mri:additionalDocumentation/cit:CI_Citation }\end{array}$ \\
\hline $\begin{array}{l}\text { software } \\
\text { Help }\end{array}$ & CreativeWork & Software application help. & identificationInfo.additionalDocumentation* & $\begin{array}{l}\text { /mdb:MD_Metadata/mdb:identificationlnfo/*/ } \\
\text { mri:additionalDocumentation/cit:CI_Citation }\end{array}$ \\
\hline $\begin{array}{l}\text { software } \\
\text { Requiremen } \\
\text { ts }\end{array}$ & $\begin{array}{l}\text { SoftwareSourceC } \\
\text { ode }\end{array}$ & Required software dependencies & identificationInfo.additionalDocumentation* & $\begin{array}{l}\text { /mdb:MD_Metadata/mdb:identificationlnfo/*/ } \\
\text { mri:additionalDocumentation/cit:CI_Citation }\end{array}$ \\
\hline $\begin{array}{l}\text { software } \\
\text { Version }\end{array}$ & Text & Version of the software instance. & identificationInfo.citation.edition & $\begin{array}{l}\text { /mdb:MD_Metadata/mdb:identificationlnfo/mri } \\
\text { :MD_Dataldentification/mri:citation/cit:CI_Citati } \\
\text { on/cit:edition }\end{array}$ \\
\hline $\begin{array}{l}\text { storage } \\
\text { Requiremen } \\
\text { ts }\end{array}$ & Text or URL & Storage requirements (free space required). & identificationInfo.environmentDescription* & $\begin{array}{l}\text { /mdb:MD_Metadata/mdb:identification Info/mri } \\
: \text { MD_Dataldentification/mri:environmentDescrip } \\
\text { tion/gco:CharacterString }\end{array}$ \\
\hline $\begin{array}{l}\text { supporting } \\
\text { Data }\end{array}$ & DataFeed & Supporting data for a Software Application. & identificationInfo.associatedResource.name* & $\begin{array}{l}\text { /mdb:MD_Metadata/mdb:identificationlnfo/*/ } \\
\text { mri:associatedResource/mri:MD_AssociatedRes } \\
\text { ource/mri:name/cit:Cl_Citation }\end{array}$ \\
\hline
\end{tabular}


2 Multiple codemeta terms are mapped to this ISO XML element, some with different attributes 